\title{
Loupe Magnification for Small Incision Cataract Surgery - an Alternative to Microscope Magnification?
}

\author{
Singh SK, ${ }^{1}$ Winter $\mathrm{I}^{2}$ Hennig $\mathrm{A}^{3}$ \\ ${ }^{1}$ Medical Director, Biratnagar Eye Hospital, Biratnagar, Nepal, ${ }^{2}$ Vitreoretinal Consultant, Biratnagar Eye Hospital, Biratnagar, ${ }^{3}$ Pro- \\ gramme Director, Sagarmatha Chaudhary Eye Hospital, Lahan, Nepal
}

\section{ABSTRACT}

A Prospective randomized controlled study was conducted to compare outcome of Small Incision Cataract Surgery (SICS) using microscope or loupe magnification.

Two hundred fifty one patient with mature cataract were randomly allocated to SICS-Fishhook Technique with either microscope (127 eyes) or loupe (124 eyes) magnification. Intra- and postoperative complications and immediate visual outcome were analyzed.

Nearly two third (microscope 65\% and magnifying loupe 62.9\%) of all patients had good visual outcome on first postoperative day. Poor outcome $(<6 / 60)$ was recorded in $8 \%$ (microscope group) and $7 \%$ (magnifying loupe group). Mean visual acuity with Snellen was 0.39 (SD 0.2) in microscope group and 0.38 (SD 0.2) in magnifying loupe group. Intra operative complications were comparable in both groups. Mean surgery time with loupe magnification was significantly shorter.

Comparatively equivalent good surgical outcome was achieved with loupe as well as with microscope magnification. However performing SICS with loupe magnification is significantly faster. Small incision cataract surgery with loupe magnification is safe and effective procedure for cataract surgery so it can play a role in reducing cataract blindness in developing countries of the world.

Key words: loupe, magnification, microscopic, cataract, surgery

\section{INTRODUCTION}

Cataract is the leading cause of blindness in the world causing more than 18 million bilateral blindness. ${ }^{1-3}$ Most of these blind people reside in developing countries. ${ }^{4}$ Ophthalmologists working in rural areas of a developing country face a Herculean task to deal with huge backlog of cataract blindness.

With the changes in cataract surgical techniques during the last decade's surgeons require a higher magnification to perform good quality extracapsular procedures with in-the-bag intraocular lens insertion. High volume SICS has become an accepted surgical technique with good visual outcome to deal with the cataract backlog in developing countries. ${ }^{5-7}$ Microscope magnification is nowadays widely used for cataract surgery, whereas few centres use high quality prism loupes to perform high volume SICS. ${ }^{6,8}$ This is the first study to determine whether loupe magnification is an alternative to microscope magnification for performing SICS. 
Singh et al. Loupe Magnification for small Incision Cataract Surgery-an Alternative to microscope magnification?

\section{MATERIAL AND METHODS}

A prospective hospital based randomized study was conducted at Biratnagar Eye Hospital (BEH), Nepal, for a period of one month (13 March 2007 to 12 April 2007). Verbal consent was taken from the patients.

Mature senile cataracts (white or brown) were included for this study. All patients underwent slit lamp examination. Immature cataract, complicated cataract, congenital cataract, developmental cataract and cataract associated with other diseases were excluded from this study.

Patients with mature senile cataracts were divided into two groups before receiving retrobulbar anaesthesia. Randomization was done with the help of random number tables. Patients with odd numbers were selected for microscope magnification and even numbers were selected for loupe magnification. All patients were operated by a single surgeon (SKS).

As the operating surgeon was the only ophthalmologist available at $\mathrm{BEH}$ at the time of this study, masking did not seem practical. All cases were operated and examined in the postoperative period by him. the visual acuity recording person was not aware of the study and masking could be achieved.

A hand-held Auto Refract Keratometer (NIKON Retinomax K plus 2) was used for keratometry and $A$ Scan ultrasound machine (Nidek ECHO scan US 800) for the purpose of axial length measurement. The power of the intra ocular Lens was calculated with the modified SRK II formula.

After pupil dilatation with Tropicamide and Phenylephrine eye drops a retrobulbar injection was given in sitting position and the patient requested to press the eye ball with the palm of the right hand to soften the eyeball. Preoperative povidone iodine $10 \%$ solution was used for disinfection of the periocular skin area.

The surgeon performed the operation in either sitting position on two tables with microscope or standing position on three tables with a magnifying loupe. Part of the surgical steps such as fornix based conjunctival flap and cauterization of bleeding vessels were performed by an operation theatre assistant in order to help optimizing the surgical time.

Carl Zeiss microscope (OPMI - 1 FR) or high quality Carl Zeiss Prism loupe with 5 times magnification and 300 $\mathrm{mm}$ working distance in combination with a halogen Spot light were used for the purpose of magnification. Frown shaped scleral incision, sclerocorneal tunnel, anterior chamber entry and linear capsulotomy were made with a $3 \mathrm{~mm}$ diamond keratome. Hydrodissection was done and superior equator of the nucleus was lifted from the capsular bag. Following the injection of viscoelastics behind the lens nucleus into capsular bag the lens nucleus could then be delivered with fish hook technique. With a Simcoe cannula the remaining cortex was aspirated. PMMA posterior chamber intraocular lens was implanted and anterior chamber was filled with ringer lactate solution. The operation was completed with an intracameral injection of cefuroxime. The surgical time was measured from the preparation of the sclerocorneal incision to the end of the intracameral cefuroxime injection.

Study variables included surgeon's time, intraoperative and postoperative complications and postoperative uncorrected visual acuity on first postoperative day. Postoperative uncorrected visual acuity was taken with Snellen chart at a 6 meters distance and for the purpose of calculation was converted into decimel figure.

\section{RESULTS}

Two hundred fifty one patients consented for the study, 127 were operated under microscope (Group A) and 124 with magnifying loupe (Group B).

Baseline characteristics were similar in both groups. In group A $83.5 \%$ (106 out of 127) had vision of hand movement while the remaining patients had visual acuity of 0.03 . In group B 80\% (99 out of 124) had vision of hand movement and remaining had visual acuity of 0.03 .

Mean intraocular lens power was similar in both groups. Patients from group A had 22.25 D (SD 1.24) and from group $B$ had 22.29 D (SD 1.29). Posterior capsule rupture with vitreous loss occurred in four patients from group $A$ and one patient from group $B$. Inadvertent intracapsular cataract extraction occurred in one patient from group A. All patients with posterior capsule rupture and intracapsular cataract extraction underwent anterior vitrectomy and had intraocular lens implantation. In group B, one patient had premature entry and another had wound gap and one suture was applied in both these patients to secure the wound.

Mean time spent by the surgeon per surgery was 4 minute 29 seconds in group $A$ and 3 minute 50 seconds in group B. In group A $28.3 \%$ patients and in group B $68.5 \%$ patients had surgery time of less than four minutes $(P$ value $<0.00003)$.

Postoperative complications were noticed among nine patients in group $A$ and six patients in group $B$. One patient each from both group had to undergo surgical revision on second postoperative day for PCIOL repositioning. Hyphaema (4 patients), increased anterior chamber reactions (2 patients), corneal edema 
Singh et al. Loupe Magnification for small Incision Cataract Surgery-an Alternative to microscope magnification?

(2 patients), air bubble in anterior chamber (1 patients), posterior capsule opacification (1 patient) and retinitis pigmentosa (1 patient) were responsible for poor visual acuity on first postoperative day in the group $A$. In group B hyphaema (1 patient), corneal edema (4 patients) and age related macular degeneration (1 patient) were responsible for poor visual outcome. All patients but retinitis pigmentosa ( 1 patient) and age related macular degeneration (1 patient) recovered with good outcome at the time of discharge.

Postoperatively uncorrected visual acuity was measured on the first postoperative day at 7 a.m. immediately after removal of the eye pad. In both groups nearly two third (group A $65 \%$ and group B $62.9 \%$ ) of patients had good visual outcome (6/6-6/18) ( $P$ value 0.3669). Mean visual acuity was 0.39 (SD 0.2 ) in group $A$ and 0.38 (SD 0.2) in group B. Poor outcome (Unaided visual acuity $<6 / 60$ ) was noticed in $8 \%$ patients from group $A$ and $7 \%$ patients from group $B$.

\section{DISCUSSION}

Preoperatively nearly all patients were blind in both groups (group A $97 \%$ blind and group B $98 \%$ blind). On day one postoperatively, $92 \%$ of patients had uncorrected visual acuity better than $6 / 60$ in group $A$ and $93 \%$ in group B. Similarly both groups (microscope $65 \%$ vs. magnifying loupe $63 \%$ ) had comparable good visual outcome better than $6 / 18$ (P value 0.3669 ). Only one patient from both groups had poor visual acuity at the time of discharge.

Hennig et al in their prospective study achieved good visual outcome in $76.8 \%$ operated cases with loupe magnification at the time of discharge. ${ }^{8}$ Another study of small incision cataract surgery with microscope showed that only $47.9 \%$ of eyes obtained uncorrected visual acuity of $6 / 18$ or better at 6 weeks. ${ }^{9}$

Intraoperative complications were comparable in both groups. There were five cases of intraoperative complications in group A vs. three cases in group B. Four posterior capsule breaks with vitreous loss occurred in the group A whereas only one in the group $B$.

Surgical time included all surgical steps performed by the surgeon during the surgery. It did not include the time taken by ophthalmic paramedics for the preparation of operating eyes. With magnifying loupe, surgeries were performed on three tables and with microscope surgeries were performed on two tables. While surgeon was carrying out surgery on one patient, ophthalmic paramedics cleaned the periocular area, draped the eye, prepared the conjunctival flap and cauterized the bleeding vessels of other patients. More patients from the group $B(68.5 \%)$ had mean surgical-time less than 4 minutes compared to $28.3 \%$ in group $A(P$ value $<0.00003$ )
Surgeries with magnifying loupe were performed in standing position whereas with microscope the sitting position was more practical. It is easier for the operating surgeon and the assistant likewise to move from one table to another in standing position. Lesser magnification, flexibility in judging intraocular structures from different angles during surgery, and probably lower number of posterior capsule rupture with vitreous loss resulted in a shorter mean surgical time with magnifying loupe ( 3 minute 50 seconds) compared to microscope (4 minute 29 seconds). Change in head posture of patient during surgery requires vertical and horizontal adjustment of optical part of the microscope to remain in good focus of the operation site whereas with the magnifying loupe the surgeon can adjust immediately by just moving his head as required. These results in a shorter time needed for focus adjustment with loupe magnification. Venkatesh et al from a high volume set up of SICS at Aravind Eye Hospital reported less than 4 minutes surgical time per case with microscope. ${ }^{7}$ In this prospective study, twelve surgeries per hour could be performed with microscope whereas up to eighteen surgeries per hour could be performed with magnifying loupe. Ruit et al reported 8-10 cases per hour with microscope using single long table. ${ }^{10}$

A good quality operating microscope is expensive equipment for many private eye clinics and eye units in this part of the world. Compared to Zeiss operating microscope, Zeiss magnifying loupe is much cheaper, easy to transport and almost maintenance free. However an external high quality light source is needed to work with the Zeiss magnifying loupe. Cost of the Zeiss magnifying loupe used for this surgery was Euro 700 and the one halogen illumination (Hanalaux) used for this surgery has a price of Euro 1500. This adds up to Euro 2200 for equipping a surgeon during SICS with magnifying loupe. However good quality light sources manufactured in India may be bought cheaper and will decrease the total cost involved for surgery with magnifying loupe. In comparison OPMI-1 FR used for this study was purchased for Euro 7200. Introduction of high quality Zeiss magnifying loupe in the operation theatre of eye units for small incision cataract surgery will increase the efficacy of ophthalmic surgeons without compromising the surgical quality and without increasing the cost.

Blind people living in hilly and mountainous areas have difficulties in accessing eye care services because of difficult terrain. ${ }^{11}$ Surgical eye camps are therefore still common and acceptable means of reducing the burden of cataract blindness in these communities. ${ }^{12}$ It is always a difficult task to carry a portable microscope and generator along with other necessary instruments and disposables for cataract surgery to these areas. Surgery with high quality Zeiss magnifying loupe could be an 
Singh et al. Loupe Magnification for small Incision Cataract Surgery-an Alternative to microscope magnification?

excellent and reliable replacement for cataract surgery with operating microscope on cataract blind patients in remote hilly areas and in other remote places.

In past times a magnifying loupe was used to improve the visibility and safety during intracapsular cataract extraction procedures. With the shift to extracapsular cataract extractions operating microscope was used routinely for the purpose of magnification. In developing countries where largest numbers of cataract blind patients live, ophthalmic surgeons are trained to operate with microscope during their residency and fellowship programs and instinctively believe that cataract surgeries done under microscopic magnification yield a better outcome.tFindings of this study clearly show that for small incision cataract surgeries, high quality magnifying loupe is as effective as the good operating microscope and at the same time surgeries can be performed faster. In a high volume surgical set up, introduction of high quality loupe magnification instead of microscope magnification will increase the numbers of cataract surgeries without increasing the number of cataract surgeons.
In plains areas of Nepal all eye hospitals have increased number of patients in the busy periods (October to March). Introduction of loupe magnification will help to raise the number of cataract surgeries performed by these hospitals. Improved efficiency of cataract surgeons with higher surgical output from these hospitals may help in adjusting the fee structure of cataract surgeries, thus making it more affordable to the poorer people in need living in developing part of the world.

\section{CONCLUSION}

Small incision cataract surgeries were performed faster with equally good visual outcome and comparative complication rate under high quality magnifying loupe magnification. In developing countries where cataract blindness is a major cause of avoidable blindness, small incision cataract surgery with high quality magnifying loupe could be an appropriate and more universal skill for the reduction of cataract blindness. A high quality magnifying loupe is a good alternative to the operating microscope and provides similar surgical outcome with increased output.

Table 1. Baseline characteristics

\begin{tabular}{llll}
\hline & Microscope (Group A) & Magnifying loupe (Group B) & P value \\
\hline Age (Mean +/- SD) & 62.4 (SD 11.9) yrs & 62.7 ( SD 11.7) yrs & 0.4364 \\
Male & $48 \%$ & $47 \%$ & 0.2389 \\
VA (HM or worse) & $83.5 \%$ & $80 \%$ & \\
Mean VA (remaining patients) & 0.03 (SD 0.02) & 0.03 (SD 0.05) & \\
\hline
\end{tabular}

Table 2. Intraoperative findings

\begin{tabular}{|c|c|c|c|}
\hline & Microscope (Group A) & Magnifying loupe (Group B) & \\
\hline Mean IOL power & $22.25 \mathrm{D}(\mathrm{SD} 1.24)$ & $22.29 \mathrm{D}(\mathrm{SD} 1.29)$ & \\
\hline \multirow[t]{3}{*}{ Intraoperative complications } & PCR + vitreous loss: 4 & PCR + vitreous loss: 1 & \\
\hline & ICCE: 1 & Premature entry: 1 & \\
\hline & & Wound gap: 1 & \\
\hline Mean surgery time $>4$ min & $71.7 \%$ & $31.5 \%$ & $P$ value $<0.00003$ \\
\hline
\end{tabular}

\section{REFERENCES}

1. Sapkota YD, et al. Prevalence of blindness and cataract surgery in Gandaki Zone, Nepal. Br J Ophthalmol 2006;90(4):411-6.

2. Foster, A. and S. Resnikoff, The impact of Vision 2020 on global blindness. Eye 2005:19(10):1133-5.

3. Ceklic, L., S. Latinovic, and P. Aleksic, [Cataract as a leading cause of visual disability and blindness in the region of Eastern Sarajevo and Eastern Herzegovina]. Med Pregl, 2005;58(9-10):449-52.
4. Ladnyi ID, Thylefors B. World Health Organization's programme on the prevention of blindness. J Hyg Epidemiol Microbiol Immunol 1983;27(4):365-71.

5. Ruit $S$, et al. An innovation in developing world cataract surgery: sutureless extracapsular cataract extraction with intraocular lens implantation. Clin Experiment Ophthalmol 2000;28(4):274-9. 
Singh et al. Loupe Magnification for small Incision Cataract Surgery-an Alternative to microscope magnification?

6. Hennig, A., High volume cataract surgery at Lahan Eye Hospital, Nepal Management, Outcome and Cost. AsiaPacific Journal of Ophthalmology 2003;15(4):9-11.

7. Venkatesh R, et al. Outcomes of high volume cataract surgeries in a developing country. Br J Ophthalmol 2005,89(9):1079-83.

8. Hennig A, et al. Sutureless cataract surgery with nucleus extraction: outcome of a prospective study in Nepal. Br J Ophthalmol 2003;87(3):266-70.

9. Gogate PM, et al. Extracapsular cataract surgery compared with manual small incision cataract surgery in community eye care setting in western India: a randomised controlled trial. Br J Ophthalmol 2003;87(6):667-72.
10. Ruit $\mathrm{S}$, et al. A prospective randomized clinical trial of phacoemulsification vs manual sutureless small-incision extracapsular cataract surgery in Nepal. Am J Ophthalmol 2007;143(1):32-8.

11. Sapkota YD, et al. Barriers to up take cataract surgery in Gandaki Zone, Nepal. Kathmandu Univ Med J (KUMJ) 2004;2(2):103-12.

12. Ruit $\mathrm{S}$, et al. Low-cost high-volume extracapsular cataract extraction with posterior chamber intraocular lens implantation in Nepal. Ophthalmology 1999;106(10):1887-92. 